\title{
Technology to Facilitate Telehealth in Applied Behavior Analysis
}

\author{
Kimberly A. Zoder-Martell ${ }^{1}$
}

Andrew M. Markelz ${ }^{1}$

Margaret T. Floress ${ }^{2}$

Heather A. Skriba ${ }^{1}$

Laila Sayyah ${ }^{1}$

Ball State University ${ }^{1}, 2000$ W. University Ave, Muncie, IN 43036

Eastern Illinois University ${ }^{2}, 600$ Lincoln Avenue Charleston, IL 61920

Corresponding Author, kamartell@bsu.edu, 2000 W. University Ave, Muncie, IN 43036, USA,

Phone: 765-285-5700 


\section{Technology to Facilitate Telehealth in Applied Behavior Analysis}

\section{Editor's Note}

This manuscript is being published on an expedited basis, as part of a series of emergency publications designed to help practitioners of applied behavior analysis take immediate action to adjust to and mitigate the COVID-19 crisis. This article was submitted on 4/21/20 and received final acceptance on $05 / 05 / 20$. The journal would like to especially thank Dr. Michael Cameron for his expeditious review of the manuscript. The views and strategies suggested by the articles in this series do not represent the positions of the Association for Behavior Analysis, International or Springer Nature.

\section{Introduction}

Traditionally, center-based Applied Behavior Analysis (ABA) therapy is delivered in a face-to-face format across several sessions; however, given the recent COVID-19 pandemic, many behavior analysts practicing in ABA centers have temporarily been authorized to provide services using a telehealth model. This rapid change in service delivery has posed unique challenges for behavior analysts and families with loved ones who were receiving ABA services in centers or schools. Among one of the most pressing concerns is that behavior analysts commonly rely on direct observation of behavior to make data-based decisions regarding client treatment plans. The shift to a telehealth model in light of the COVID-19 pandemic requires behavior analysts to quickly adapt the manner in which many have been providing services. Although many behavior analysts may be familiar with various technologies, they may struggle to identify the benefits and limitations of each technology in terms of providing telehealth. Selecting an appropriate technology that allows behavior analysts to stay true to the science of behavior analysis is prudent. The purpose of this paper is to provide a review of some available 
technologies to support telehealth that will allow behavior analysts to conduct direct observation from a remote location. The three technologies reviewed are 1) web camera, 2) Swivl ${ }^{\mathrm{TM}}$, and 3) telepresence robot. Features of these technologies will be described and compared including benefits and drawbacks of each (see Table 1). Sample task analyses for using each technology are also provided.

\section{Telehealth Research}

Telehealth is a model of service delivery that utilizes telecommunications and information technology to provide access to health, assessment, diagnosis, intervention, consultation, supervision, and education (Nickelson, 1998). Although more commonly associated with psychiatric and psychological services (Vandenbos \& Williams, 2000), telehealth is a growing area of research in the fields of education and ABA. Ferguson and colleagues (2018) conducted a systematic review of telehealth interventions for 28 individuals with autism spectrum disorder. Intervention characteristics employed across included telehealth studies were functional analyses, naturalistic teachings, behavior supports, preference assessments, and comprehensive training packages about behavior analytic principles (Ferguson et al., 2018). Given positive outcomes across participants and studies, results from the review suggested that telehealth can be an acceptable platform for behavior analytic interventions and assessments. Information regarding the type of technology used to conduct the telehealth interventions, however, was limited. Only a small number of studies provided extensive information describing the technology. Of those studies, the most common technology used was video conferencing software with webcams to provide didactic trainings and video coaching (Ferguson et al., 2018).

In one study, Wacker et al. (2013) conducted functional analyses via telehealth with 20 children between the ages of 19 and 80 months who displayed problem behavior. Using 
telehealth software, the behavior consultant sat at a computer with a web cam that received video and audio from a regional pediatric clinic located miles away. The behavior consultant was able to remotely move the camera up, down, and side to side so that the parent and child remained visible at all times. The behavior consultant trained parents via teleconferencing about the study's purpose and procedures and also interviewed the parent about their child's problem behaviors. Lastly, the behavior consultant coached and supported the parent in conducting a functional analysis to identify the maintaining variable of the problem behavior. For 18 out of 20 participants, the behavior consultant identified environmental variables as the maintaining variable for problem behaviors with interrater agreement over $90 \%$. Results from the study suggested that behavior consultants can collaborate with parents and conduct functional analyses effectively and efficiently via telehealth.

\section{Telehealth Technologies}

Even though telehealth has been the subject of research for decades with some early work involving the use of telephones to support parent trainings (e.g., Patterson 1974) or 'bug in ear' technology to provide real-time coaching (e.g., Bowles \& Nelson, 1976), exponential advancements in technology have provided a range in available technologies to support telehealth from low tech to high tech. Despite research in support of telehealth, many behavior analysts may express some hesitation using telehealth technologies due to an unfamiliarity with the available technologies. In the following sections, we outline three technologies (i.e., webcam, Swivl, telepresence robots) that behavior analysts can select to use when implementing ABA services via a telehealth model that will allow for direct observation as well as uses for videoconferencing software. We conclude with a brief discussion of ethical considerations as well general tips for using telehealth with a family for the first time. 
Web camera. Web cameras are commonplace today and are a standard feature on many laptop and desktop monitors. The web camera uses videoconferencing software to transmit the video in real time. If an older laptop or desktop screen is still in use, cost effective clip-on web cameras can be installed with relative ease (see Table 1). Clip on web cameras can plug into a computer, but others are wireless and use WIFI. Headphones and an external microphone are sometimes used to enhance sound quality. There are a number of available features for web cameras including built-in microphones, lights to notify the user when the web camera is on, tilting/panning, or autofocus. When selecting an external web camera, these features should be considered. External web cameras are inexpensive and accessible to most users. In fact, the most basic web cameras can be purchased for approximately twenty dollars.

Web cameras are the most easily accessible of the technologies reviewed. They are built into most devices (e.g., laptops, cell phones, tablets) and are inexpensive to purchase independently as a clip-on device. There is very minimal set-up required for web cameras. Initial set-up generally comprises of downloading the desired application and allowing it to access the computer's microphone and camera. After this, accessing the web camera's basic functions becomes automated upon use. A benefit to purchasing an external web camera is the ability to position the camera to obtain a better view of the environment, as opposed to a fixed internal web camera that is positioned to capture the computer user's face.

A notable limitation of web camera's is a behavior analyst's inability to fully immerse in their client's environment. The web camera provides a limited view of the environment. The quality of picture and sound is impacted by internet connection qualities. Furthermore, an external microphone may need to be purchased. Additionally, external cameras can be confusing to clip on and navigate during early use. 
Tips for use. A sample task analysis for using a Logitech ${ }^{\mathrm{TM}}$ web camera is provided (Table 2). When selecting an external web camera, behavior analysts should suggest a camera that has an indicator light letting clients know when they are being recorded. The consultee (e.g., parent, client) should be provided with training for turning on/off the camera and operating software. At the end of each session ensure that the camera is turned off to protect privacy. Clients may choose to place a small piece of painter's tape over the camera lens. If using a videoconferencing application that provides clinician's one universal meeting link, be sure to "lock" the meeting when all parties are present.

Research support. A number of studies have been conducted in which web-cameras were used. In one study conducted by Simacek et al. (2017), the researchers utilized web cameras for training and coaching caretakers on the implementation of a functional communication intervention for three children with developmental disabilities. Functional assessments were also conducted virtually prior to the intervention, and all three children acquired the communication responses targeted in the study. Results indicated caretakers were able to implement the intervention successfully across various settings. Furthermore, caretakers rated the treatment as acceptable.

Swivl. A Swivl (https://www.swivl.com/) is a video consultation device with the ability to rotate 360 degrees in order to follow and record the presenter with a connected tablet (e.g., iPad). The presenter wears a transmitter on a lanyard which emits a signal to the robot to notify it of the wearer's movement (Swivl, n.d.). The device also serves as a stand for the tablet when used as the recording device. A microphone is built into the transmitter to record the presenter's speech, and additional transmitters can be synched to the device to record more than one individual (Carson et al., 2007). Real-time video can be streamed with other users by using Swivl 
in tandem with a video conference application (Swivl, n.d.). After recording, videos can be accessed through the Swivl application on the tablet, as well as through the Swivl website on a computer. Users are then able to review, annotate, and ask questions about the recorded sessions. There are several benefits for behavior analysts choosing to use a Swivl for telehealth. The Swivl is a good option for telehealth when the behavior analyst wants to directly observe the client in the environment. One of the most notable benefits is the ability of the Swivl to move 360 degrees. With the use of the transmitter lanyard, the camera does not need to be manually readjusted. Furthermore, the transmitter lanyard includes a microphone, which greatly improves the audio quality. There are also several video options available. ABA therapy sessions can be conducted using the live feed option, or sessions can be video recorded and viewed at a later time by the behavior analysts. The ability to annotate sessions is unique to the Swivl and may be an option for tech-savvy parents who would benefit from feedback.

Compared to webcams, the Swivl may be cost prohibitive. The device is more expensive than a webcam and requires they use of an external tablet that may also need to be purchased. Despite the cost, the Swivl may be a good long-term purchase from ABA providers as it has utility for supervision, home-based therapy, and clinic-based interventions. Another potential limitation of the Swivl is the use of the transmitter lanyard. Although parents would likely be comfortable wearing the lanyard, an individual with autism spectrum disorder may refuse. Finally, signals between the transmitter and the device can be easily obstructed when the individual with the transmitter lanyard moves out of range or due to internet connectivity issues. These issues may impact sound and video quality.

Tips for use. A task analysis for setting up the Swivl is provided (Table 3). The Swivl should be set up in a high trafficked area where a 360-degree view is possible. It is best to set the 
Swivl up on a table that is toward the middle of the room. One important consideration is to determine the best person to wear the lanyard. For example, if the behavior analyst would like to observe the client's behaviors the client should wear the lanyard. If observing the client is not as important for the session, the parent can wear the lanyard. When using the Swivl, sound quality in noisy settings may be jeopardized. Behavior analysts should be aware that the Swivl (n.d.) provides users with set up suggestions in order to maintain video and audio quality of recordings, and the microphone has features to help user minimize background noise in recordings.

Research support. The Swivl has been used in a number of studies (e.g., Johnson et al., 2019; McCoy et al., 2018). Most of the available studies have evaluated the use of a Swivl in school-based research. In a pilot study conducted by Franklin et al. (2018), researchers recruited teachers from public schools to set up and use a Swivl in their classrooms and report strengths and challenges found when using the technology. Participants noted ease of use, both in setting up the device and accessing recordings; however, there were reported issues with internet connectivity.

Telepresence robot. A telepresence robot is a videoconferencing screen (e.g., tablet) that is mounted on a wheeled-base that can be controlled from a remote location allowing for a more physical virtual presence than the two other technologies described. There are a variety of vendors that sell telepresence robots. Of the three technologies, telepresence robots can have the highest cost. The most basic telepresence robots are similar in cost to the Swivl; however, some sell for up to $\$ 30,000$. The available features vary greatly depending on the cost; however, for purposes of behavior analytic telehealth, the more basic models are likely adequate. Double Robotics $^{\mathrm{TM}}$ (n.d.) offers amongst the more affordable telepresence robots. 
The primary benefit of the telepresence robot is the ability of the behavior analyst to have a more physical virtual presence. The behavior analyst can control the robot from a remote location and can navigate through the environment. This physical presence may be helpful for developing and maintain rapport with parents. Furthermore, the ability to navigate through the environment may be especially useful in high stakes or high-risk cases. Although the telepresence robot is the most expensive option, it may be a good long-term investment for ABA centers, especially those centers that plan to continue telehealth or those that provide supervision to therapists providing home-based and school-based therapy. The supervising behavior analyst can provide feedback and training without leaving the ABA clinic. The telepresence robot requires the use of a secure app where data are encrypted. Third party video-conferencing software is not required. Finally, families clearly determine when the behavior analyst can be virtually present as the client is required to login to the app in order for the behavior analyst to navigate the robot in the environment.

There are several potential limitations for using Telepresence robots. Most notably is the cost of the telepresence robot. Given the cost, this may only be an option for practitioners that already have telepresence robots. The telepresence robot is initially more complicated to set up; however, once it is set up, parents only need to login to the tablet. The telepresence robot typically requires a strong high-speed internet connection, which may not be available in certain communities (e.g., rural). The telepresence robot must be plugged into an electrical outlet to maintain a battery charge when not in use. Finally, the telepresence robot is more obtrusive than the other technologies and clients may be more reactive to the technology.

Tips for use. Parents should be provided with a task analysis to set up the telepresence robot. A sample task analysis for setting up the Double Robotics Duo $2^{\mathrm{TM}}$ telepresence robot is 
provided (Table 4). The use a remote control (e.g., PS4 ${ }^{\mathrm{TM}}$ controller), as opposed to the keys on the computer, may help with navigation. The behavior analyst should "test-drive" the telepresence robot before using the robot in homes. Although the robot is not necessarily difficult to navigate, it does require some practice. Consider the goals of the therapy session and reserve the use of a telepresence robot for high needs cases.

Research support. Fischer, Bloom, et al. (2018) conducted a study to evaluate how threestep prompting impacted student compliance in special education classrooms. Two telepresence robots were used and operated remotely by consultants and research assistants to observe the environment. The researchers found that telepresence robot problem-solving teleconsultation was an effective means of increasing compliance, had high levels of acceptability for all participants, and moderate levels of treatment integrity. Teacher participants reported that the telepresence robot was an acceptable manner by which consultation services were provided.

\section{Videoconferencing Software}

Many of the technologies that are used for telehealth require the use of third-party videoconferencing software. There are a range of available videoconferencing products and each have different features (e.g., encryption, ability to record). Videoconferencing software should be selected based on the user's needs. Fischer, Shultz, et al. (2018) reviewed videoconferencing technology that has been cited in the school consultation literature. Features of the technology were reviewed including the cost, computer compatibility, number users, onscreen document sharing, integrated cloud storage, instant messaging, and compliance with the Health Insurance Portability and Accountability Act and Family Educational Rights and Privacy Act (HIPAA, 1996; FERPA, 1974). As with using any online platform, there are ethical considerations related to confidentiality. To the extent possible, behavior analysts should select platforms that are 
compliant with HIPAA and FERPA. For a discussion of the ethical considerations for using teleconsultation, readers should review Fischer et al. (2019).

\section{Ethical Considerations}

Traditionally, behavior analysts have not delivered services to clients using a telehealth model as managed healthcare providers require direct contact with the client. Furthermore, much center-based ABA occurs in a 1:1 or small group format. Therefore, telehealth is a new treatment modality for many behavior analysts. Regardless of the technology selected, there are a number of ethical considerations for implementing ABA therapy via a telehealth model that are outside of the scope of this paper. Behavior analysts are encouraged to read Cox et al. (2020) for a discussion of ethical considerations related to providing ABA services given COVID-19. Behavior analysts are further encouraged to seek out training opportunities for using a telehealth model. A number of providers are currently offering online training for using a telehealth model.

\section{General Telehealth Tips}

In addition to selecting a technology to support telehealth, there are a number of additional considerations that behavior analysts may find helpful. The following strategies are suggested when using telehealth for the first time, regardless of the technology selected.

1. Obtain informed consent for using telehealth.

2. Consider privacy and confidentiality. Advise parents of the limitations to privacy and confidentiality when using the Internet.

3. Assess the family's comfort and familiarity with technology.

4. Introduce technology the family to the technology via a phone conversation.

5. Provide a video and written instructions to set up technology.

6. Keep the technology set up in the home to manage reactivity. 
7. Check-in with parent/client to make sure it is a good time to observe prior to turning on the technology.

\section{Summary}

Given the recent COVID-19 pandemic, many behavior analysts are temporarily transitioning from school and center-based ABA therapy services to using a telehealth model. Although telehealth is widely used in other fields (e.g., medicine), it is less practiced in the field of behavior analysis. As a result, behavior analysts have needed to rapidly transition to a model of service delivery in which many have little experience. As behavior analysts transition to using telehealth to support clients during COVID-19, there are a number of considerations, including the selection of technology. Behavior analysts may find the use of webcams, Swivls, or telepresence robots useful in facilitating telehealth. 


\section{References}

Bowles, P. E., \& Nelson, R. O. (1976). Training teachers as mediators: Efficacy of a workshop versus the bug-in-the-ear technique. Journal of School Psychology, 14(1), 15-26. https://doi.org/10.1016/0022-4405(76)90058-3.

Carson, C., Callard, C., Gillespie, R., Choppin, J., \& Amador, J. (2019). Bridging the distance: One-on-one video coaching supports rural teachers. The Learning Professional, 40(6), 66-70.

Cox, D. J., Plavnick, J., \& Brodhead, M. T. (2020, March 31). A Proposed Process for Risk Mitigation During the COVID-19 Pandemic. https://doi.org/10.31234/osf.io/buetn

Double Robotics. (n.d.). Retrieved from https://www.doublerobotics.com/

Family Educational Rights and Privacy Act of 1974, 20 U.S.C. § 1232g. (1974).

Franklin, R., Mitchell, J., Walters, K., Livingston, B., Lineberger, M., Putman, C., Yarborough, R., Karges-Bone, L. (2018). Using Swivl robotic technology in teacher education preparation: A pilot study. TechTrends, 62,184-189. https://doi.org/10.1007/s11528-017$0246-5$

Fischer A., Bloomfield, B., Clark, R., McClelland, A., \& Erchul, W. (2018). Increasing student compliance with teacher instructions using telepresence robot problem-solving teleconsultation. International Journal of School \& Educational Psychology, 7, 158-172. https://doi.org/10.1080/21683603.2018.1470948

Fischer, A., Collins, T., Dart, E., Radley, K. (2019). Technology applications in school psychology consultation, supervision, and training. New York: Routledge

Fischer, A., Schultz, B., Collier-Meek, M., Zoder-Martell, K., Erchul, W. (2018) A critical review of videoconferencing software to support school consultation. International 
Journal of School \& Educational Psychology, 6(1), 12-22, doi:10.1080/21683603.2016.1240129

Health Insurance Portability and Accountability Act (HIPAA). Pub. L. No. 104-191, § 264, 110 Stat.1936. (1996).

Johnson, E. S., Zheng, Y., Crawford, A. R., \& Moylan, L. A. (2019). Developing an explicit instruction special education teacher observation rubric. The Journal of Special Education, 53(1), 28-40. https://doi.org/10.1177/0022466918796224

McCoy, S., Lynam, A., \& Kelly, M. (2018). A case for using Swivl for digital observation in an online or blended learning environment. International Journal on Innovations in Online Education, 2(2). doi:10.1615/IntJInnovOnlineEdu.2018028647

Nickelson, D. W. (1998). Telehealth and the evolving health care system: Strategic opportunities for professional psychology. Professional Psychology: Research and Practice, 29(6), 527-535. https ://doi.org/10.1037/0735-7028.29.6.527.

Oliver, R. M., \& Reschly, D. J. (2010). Special education teacher preparation in classroom management: Implications for students with emotional and behavioral disorders. Behavioral Disorders, 35(3), 188-199. doi:10.1177/01987 4291003500301

Patterson, G. R. (1974). Interventions for boys with conduct problems: Multiple settings, treatments, and criteria. Journal of Consulting and Clinical Psychology, 42(4), 471-481. https://doi.org/10.1037/h0036 731 .

Simacek, J., Dimian, A., \& McComas, J. (2017). Communication intervention for young children with severe neurodevelopmental disabilities via telehealth. Journal of Autism and Developmental Disorders, 47(3), 744-767. doi:10.1007/s10803-016-3006-z

Swivl. (n.d.). Retrieved from http://www.Swivl.com/ 
Wacker, D. P., Lee, J. F., Dalmau, Y. C. P., Kopelman, T. G., Lindgren, S. D., Kuhle, J., Pelzel, K, E., \& Waldron, D. B. (2013). Conducting functional analyses of problem behavior via telehealth. Journal of Applied Behavior Analysis, 46(1), 31-46. 
Table 1

Comparison of Telehealth Technologies

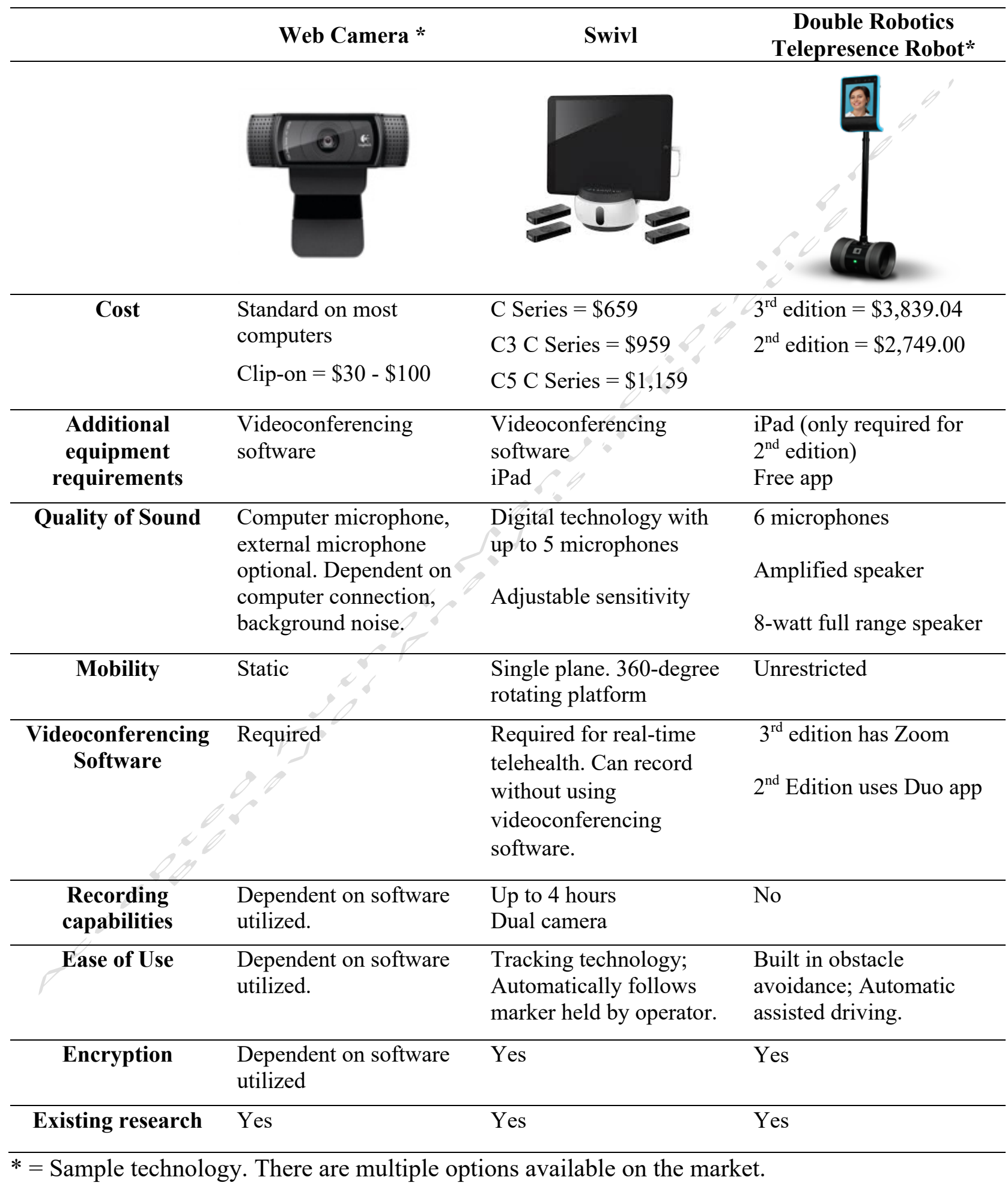


Table 2

Sample Task Analysis for Using Webcam

\section{Logitech Web Camera}

Set - Up:

1. Open Logitech box

2. Remove Logitech device

3. Pull down the flexibility clip/base of the Logitech and secure onto the top of your computer where the built-in camera generally is

4. Open Skype for Business

a. Login with your Outlook information

5. Plug in Logitech to computer's USB port

6. A pop-up should appear asking if you would like to use the Logitech as preference. Select yes.

a. If the pop-up does not appear:

i. Click "Skype for Business" in the top left corner of computer screen

ii. Select "preferences"

iii. Ensure "microphone" and "camera" is set to Logitech Webcam C930e

7. Flip open the privacy cover

8. Position Logitech camera to capture your face and/or the setting

a. Can do this by:

i. Click "Skype for Business" in the top left corner of computer screen

ii. Select "preferences"

iii. Select "audio/video"

9. Click on "contacts" to select who to call

10. Click on video camera image ("Start a video call”)

\section{Tear Down:}

1. Click the red phone symbol ("hang up")

2. Click "Skype for Business" at top left corner of computer

3. Click "Sign Out"

4. Click "Skype for Business" at top left corner of computer 
5. Click "Quit Skype for Business"

6. Close privacy cover on Logitech

7. Unplug Logitech from computer

8. Take Logitech from where it is resting on computer

9. Close the flexibility clip/base of the Logitech

10. Return Logitech to box

11. Close Logitech box. 
Table 3

Sample Task Analysis for Using Swivl

\section{Swivl}

Set - Up:

1. Unzip the SWIVL container

2. Remove white cord out and SWIVL from case and place on a flat surface

3. Insert USB on white cord into the USB port on the back side of the SWIVL

4. Connect opposite end of white cord to charging port on the iPad

5. Insert iPad into SWIVL

6. Press and hold power button on SWIVL

7. Open Swivl app

8. Remove the small remote on top of SWIVL

9. Then hold the power button on the remote for 3-4 seconds. The LEDs on the remote will light up and the bottom LED will flash red as it searches for the base. Both lights on the remote should turn green, indicating that it paired with this base. The Primary Marker is the one that the base will respond to and track.

10. SWIVL follows movement of remote through sensors on the front of the remote and the front of SWIVL. Practice moving the SWIVL with the remote

11. Tap the button on the middle left side of the screen. The word 'zoom' should pop up, click the word zoom. This will take you to a new app.

12. Select new meeting

13. Select start meeting

14. Click "Participants" in upper right corner

15. Click "Invite" this is how you would conduct a live consultation.

\section{Tear Down:}

1. Press and hold power button on SWIVL to turn off

2. Place small remote back on the SWIVL

3. Disconnect USB from iPad and SWIVL, place cord back into SWIVL container

4. Set iPad aside

5. Put SWIVL back into the case and zip shut 
Table 4

Sample Task Analysis for Using a Telepresence Robot

\section{Double Robotics Duo Telepresence Robot}

Set - Up:

1. Set the Double box down on a flat surface and undo all six tabs.

2. Remove the iPad case located in right corner of the box and set it aside. The iPad Spacer is inside the case.

3. Remove white cardboard box and set it aside. This will not be used during set up.

4. Remove audio Kit and Camera Kit and set aside.

5. Remove foam "puzzle piece" from on the middle right end of box to gain access to the telepresence robot

6. Take the Telepresence Robot out from the box and place it standing-up on a flat surface. The side with "Double" on it is the front of the robot.

7. Open Audio Kit and take it out of its box.

8. Slide the back of the audio kit off,

9. Position Audio Kit on top of the pole and place the speaker at the front with the cords facing up. Ensure the speaker is a few inches from the top of the pole

10. Slide the back portion of the Audio Kit upward along the pole so that it fits together with the front and secures the speaker.

11. Place one cord of the Audio Kit into top of pole

12. Pick up the iPad case. Inside the case is a spacer. Slide the spacer outside of iPad case

13. Insert iPad into spacer. Line up volume button with gap of spacer.

14. Set aside spacer and remove the camera kit from its box.

15. Locate the USB port inside the iPad case. Plug the USB cord on the camera kit into the USB port inside the iPad case. You will have to thread the cord through the top of the case in order to properly do this.

16. Making sure the cord is neatly tucked; you may now slide in your iPad upside down into the iPad case.

17. Plug the Camera Kit into the charging port of the iPad.

18. Insert iPad case into the robot 
19. Remove the bolt and wrench located in audio kit

20. Take the long bolt and insert it behind the iPad head through the opening at the top.

21. Tighten with the hex wrench until it is firmly in place.

22. Plug hanging cord from audio kit into the headphone jack on the iPad

\section{Pairing Bluetooth:}

1. Turn on telepresence robot by holding down power button on the bottom back side of the robot (hold down for about 3 seconds).

2. The front LED should start flashing blue, indicating that it is ready to pair with the iPad.

3. Go to your iPad's Settings $>$ Bluetooth and locate your Double under the Devices list. Tap to pair. After a few seconds, your Double should pair with the iPad.

4. If a pop-up is on the iPad asking to pair/communicate with Double, click allow

5. Open the Double app on the iPad

\section{Instructions for the Behavior Analyst:}

1. Using a device connected to $\mathrm{Wi}-\mathrm{Fi}$, open Chrome

2. Go to www.drive.doublerobotics.com

3. Login.

4. Click on the active robot.

5. Use the arrow keys to navigate the robot 